\title{
Is resistance to ischaemia of motor axons in diabetic subjects due to membrane depolarization?
}

\author{
Michael Strupp ${ }^{1}$, Hugh Bostock ${ }^{2}$, Paul Weigl ${ }^{1}$, Klaus Piwernetz ${ }^{3}$, \\ Rolf Renner ${ }^{3}$ and Peter Grafe ${ }^{1}$ \\ ${ }^{1}$ Department of Physiology, University of Munich, Munich (F.R.G.), ${ }^{2}$ Sobell Department of Neurophysiology, \\ Institute of Neurology, London (U.K.), and ${ }^{3}$ Center for Diabetes, IIIrd Department of Medicine, Klinikum \\ Bogenhausen, Munich (F.R.G.)
}

(Received 18 April, 1990)

(Accepted 6 June, 1990)

\section{SUMMARY}

The reasons for the resistance to ischaemia of peripheral nerves in diabetics are not well understood. We have now explored whether axonal depolarization underlies this phenomenon, as has previously been proposed. Resistance to ischaemia was determined by the new method of "threshold tracking". This method revealed an increase in excitability of the peroneal nerve at the popliteal fossa during ischaemia, and a decrease in excitability in the post-ischaemic period. The extent of these alterations in 28 type 1 diabetics without peripheral neuropathy showed a strong correlation with the mean blood glucose concentrations during the last $24 \mathrm{~h}$ before examination. To test whether the ischaemic resistance was related to membrane potential, we also measured axonal superexcitability in 11 selected diabetics, since it has been shown that post-spike changes in excitability depend on membrane potential. Changes in excitability of the peroneal nerve were measured in the period between 10 and $30 \mathrm{msec}$ following a conditioning supramaximal compound action potential. Under resting conditions, no differences in the post-spike superexcitability were found between controls and diabetics, despite striking differences in their responses to a 10-min pressure cuff. These observations indicate that membrane depolarization is not involved in the resistance to ischaemia of motor axons in diabetic subjects.

Correspondence to: Dr. P. Grafe, M.D., Physiologisches Institut der Universität München, Pettenkoferstrasse 12, D-8000 München 2, F.R.G.

0022-510X/90/\$03.50 ㄷ 1990 Elsevier Science Publishers B.V. (Biomedical Division) 
Key words: Diabetes; Resistance to ischaemia; Axons; Membrane potential; Superexcitability; Blood glucose concentration

\section{INTRODUCTION}

Resistance to ischaemia is a characteristic property of the peripheral nerves in diabetes mellitus. It was first described by Steiness $(1959,1961 \mathrm{a}, \mathrm{b})$ and later confirmed by many other investigators (Gregersen 1968; Horowitz and Ginsberg-Fellner 1979; reviews by Ludin and Tackmann 1984; Low 1987; Thomas and Brown 1987). Recent publications have provided good evidence that resistance to ischaemia is due to a marked increase in energy substrate stores (Jaramillo et al. 1985; Low et al. 1985; Low 1987; Shirabe et al. 1988; Parry and Kohzu 1989). An alternative hypothesis was put forward by Ritchie (1985), that membrane depolarization is responsible for the change in sensitivity to ischaemia. This was based on computer simulations, according to which the metabolic cost of impulse propagation is reduced in slightly depolarized fibres. Several mechanisms have been described which may depolarize axons in patients with diabetes. First, endoneurial hypoxia (Tuck et al. 1984; Newrick et al. 1986; Masson 1988 ) as a result of diabetic microangiopathy (Dyck 1989) may be such a factor. Secondly, a decreased uptake of $m y o$-inositol has been reported to result in a reduced activity of the $\mathrm{Na}^{+} / \mathrm{K}^{+}$pump (Greene et al. 1988). Such a diminished $\mathrm{Na}^{+} / \mathrm{K}^{+}$ -ATPase activity has been found in biochemical studies (Das et al. 1976).

We thought it important to clarify the mechanism of the abnormal resistance to ischaemia in diabetics, since this may help to gain insight into the aetiology of diabetic neuropathies. We have previously found that "threshold tracking" provides a sensitive measure of ischaemic resistance (Weigl et al. 1989), and have used the technique in this study to select a group of subjects with pronounced ischaemic resistance, and also to evaluate the relationship between ischaemic resistance and blood glucose levels. To test for an abnormal resting potential of the motor axons exhibiting ischaemic resistance, we have measured superexcitability, the transient increase in electrical excitability that follows in the wake of an action potential. Superexcitability is strongly potential dependent, and provides a useful extracellular measure of changes in membrane potential (Bostock and Grafe 1985). Our results, which indicate that an abnormality in resting potential is not involved in the ischaemic resistance, have been reported to the Deutsche Physiologische Gesellschaft (Strupp et al. 1990).

\section{METHODS}

\section{Determination of susceptibility to ischaemia}

The technique of "threshold tracking" used in the present study to determine the susceptibility to ischaemia has been described previously (Weigl et al. 1989). In brief, muscle action potentials were recorded from $\mathrm{m}$. extensor digitorum brevis. The strength 
of stimulation of the peroneal nerve in the popliteal fossa was adjusted to maintain a constant electrical response in the muscle (30\% of maximal compound action potential). The nerve was stimulated with an $0.2-\mathrm{msec}$ current pulse once/sec. An electronic feedback system automatically increased or reduced the stimulus strength, depending on whether the muscle action potential was below or above a preset voltage. The stimulus current was fed into a sample and hold circuit and recorded by a digital oscilloscope. Since the absolute stimulus currents varied between the different subjects, the digitized data were normalized to the same pre-ischaemic current level. This enabled the data to be compared with respect to percentage changes in threshold, and it also enabled us to obtain unbiased averages of data from controls and patients. Ischaemia was induced by a pressure cuff applied to the thigh $(50-70 \mathrm{~mm} \mathrm{Hg}$ above systolic pressure).

\section{Superexcitability as an indirect measure of membrane potential}

Changes in axonal excitability were explored 10,20, and $30 \mathrm{msec}$ following a conditioning, supramaximal stimulus (peroneal nerve at popliteal fossa). The test stimulus was set to produce a muscle compound action potential amplitude that was $30 \%$ of maximal. The change in the amplitude of this conditioned potential without and with a conditioning stimulus was used as a measure of the change in axonal excitability. Fig. 1 illustrates the recording protocol in a normal subject. The upper panel (Fig. 1A) shows the change in axonal excitability $10 \mathrm{msec}$ after a preceding action potential. The supramaximal conditioning stimulus, applied to the peroneal nerve at the popliteal fossa (stimulus duration $0.2 \mathrm{msec}$ ), evoked a compound muscle action potential (CMAP) at

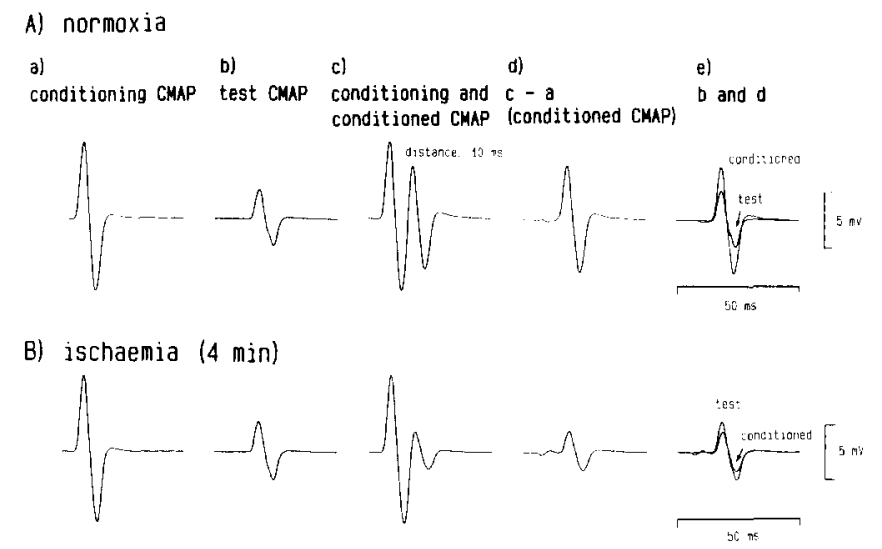

Fig. 1. Superexcitability as an indirect parameter of membrane potential. A and B show original records (average of 10 sweeps) before (A) and during (B) ischaemia ( $4 \mathrm{~min}$ ). The peroneal nerve was stimulated at the popliteal fossa (stimulus duration $0.2 \mathrm{msec}$ ); compound muscle action potentials (CMAPs) were recorded from $m$. extensor digitorum brevis. (a) Conditioning CMAP, evoked by supramaximal stimulation; (b) test CMAP, evoked by a stimulus that was adjusted to $30 \%$ of maximal CMAP; (c) response to double stimulation: conditioning CMAP followed by test stimulus ( = conditioned CMAP); (d) conditioned CMAP alone; i.e. the conditioning CMAP (trace a) was subtracted digitally from trace c; (e) the test and conditioned CMAP are superimposed. Note the bigger amplitude of the CMAP under normoxia (A) and the lack of this increase in excitability 4 min after the onset of ischaemia. 
m. extensor digitorum brevis. A test stimulus was then adjusted to evoke a CMAP of about $30 \%$ in amplitude as compared to the maximal response. For later analysis (see Fig. 4) this amplitude was defined as $100 \%$. When the test stimulus was preceded by the conditioning CMAP, an increase in amplitude was observed. This is illustrated to the right of the upper panel. There, unconditioned and conditioned CMAPs are superimposed. This phenomenon has been called superexcitability. An early description was given by Gilliat and Willison (1963). In axons, which are depolarized due to ischaemia, no post-spike superexcitability can be seen. This is illustrated in Fig. 1B. Here, the same stimulation protocol was used $4 \mathrm{~min}$ after the onset of ischaemia produced by a pressure cuff applied to the thigh. The ischaemic axon was slightly subexcitable $10 \mathrm{msec}$ after the conditioning CMAP.

\section{Controls and patients}

The mean age of 20 healthy controls ( 6 female, 14 male) was $31.3 \pm 6.7$ years (range 24-45). The patients ( 17 female, 21 male) were selected by the type of diabetes (type 1, insulin-dependent diabetes mellitus) and the absence of neuropathy. The last prerequisite was defined by (a) the absence of neuropathic symptoms or signs, (b) a normal clinical examination (reflexes and sensation), and (c) a peroneal motor conduction velocity of more than $41 \mathrm{~m} / \mathrm{sec}(\mathrm{Oh}, 1984)$. The amplitude of the compound muscle action potential from the $\mathrm{m}$. extensor digitorum brevis exceeded the lower limit of $4 \mathrm{mV}$ given by $\mathrm{Oh}$ (1984). The mean age of the patients was $31.5 \pm 12.6$ years (range 14-54). They had a mean duration of illness of $8.7 \pm 7.0$ years (range 1 month to 22 years). Their median blood glucose level within $1 \mathrm{~h}$ before and after the examination was $151 \pm 42 \mathrm{mg} / \mathrm{dl}$ (range 48-255). Capillary blood glucose concentrations were determined 3-7 times daily. From these data we calculated the mean blood glucose concentrations of the last $24 \mathrm{~h}$ and $72 \mathrm{~h}$, respectively, before examination. Mean glycosylated haemoglobin (HbAlc) of the patients was $8.8 \pm 1.7 \%$ (range 5.9-10.5) within 4 weeks before the examination.

\section{Data and statistics}

Data were recorded, stored, and analyzed on a digital oscilloscope (Nicolet 4562). Results are expressed as mean \pm SD. Since the hypotheses we tested specified the directions of possible deviations, an unpaired, one-tailed $t$-test was used to determine statistical significance. A linear regression analysis was performed to test for significant correlations between changes of excitability and blood glucose levels or HbAlc.

\section{RESULTS}

\section{Determination of susceptibility to ischaemia}

In a first series of experiments, an optimal time of ischaemia was explored for a clear difference between the ischaemic behaviour of controls and diabetics. The results are illustrated in Fig. 2. The upper panel stems from observations on several controls and diabetics on which the method of threshold tracking (see Methods; Weigl et al. 


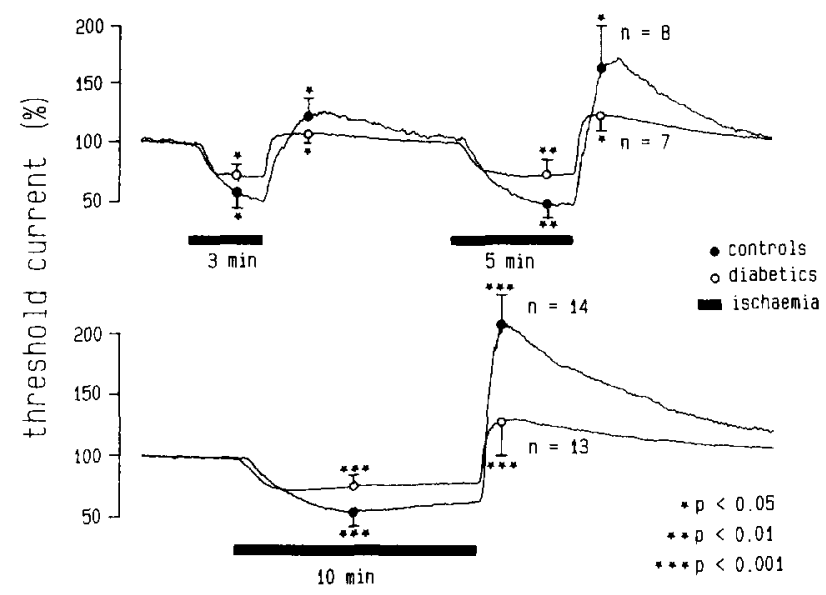

Fig. 2. Ischaemia-induced changes in excitability of human peroneal nerve in control subjects and in diabetics. The peroneal nerve was stimulated at the popliteal fossa; compound muscle action potentials were recorded from $\mathrm{m}$. extensor dig. brevis (see Methods). The data illustrated in the upper panel show the effects of a $3 \mathrm{~min}$ period of ischaemia followed by a second ischaemia of $5 \mathrm{~min}$. Results from controls and diabetics were normalized, averaged, and are superimposed (see Methods). The lower panel shows data from a second series of experiments, in which the duration of ischaemia was increased to 10 min. The differences between controls and diabetics were most obvious with the latter protocol. This protocol was, therefore, used for the following studies.

1989) was used to determine changes in excitability of the peroneal nerve during two consecutive periods of ischaemia. The first time, the pressure cuff at the thigh was at suprasystolic pressure for $3 \mathrm{~min}$; the second period of ischaemia lasted for $5 \mathrm{~min}$. The lower panel shows the average behaviour of another group of patients and controls during and after $10 \mathrm{~min}$ of ischaemia. The data show (a) $5 \mathrm{~min}$ of ischaemia are enough for the maximal difference between the ischaemic drop in threshold of controls and diabetics. After this period, a slow rise in threshold was observed in both groups. However, there was no further increase in threshold difference. (b) Differences in the post-ischaemic rise in threshold increased with the duration of ischaemia. Highly significant $(P<0.001)$ alterations were found following a $10-\mathrm{min}$ period of ischaemia. As a consequence of these observations, the longer lasting period of ischaemia was used for the comparison with superexcitability. A protocol with an ischaemic time of more than 10 min was not suitable, due to the development of pain in the leg.

Correlation between threshold changes and blood glucose levels or glycosylated haemoglobin

During the present study altogether 28 diabetics were tested by means of threshold hunting for their sensitivity to an ischaemic period of $10 \mathrm{~min}$. Changes in axonal threshold of these patients during and after ischaemia were correlated with (a) the actual blood glucose concentration, (b) the mean blood glucose concentration for the last $24 \mathrm{~h}$ before the examination, (c) the mean blood glucose concentration for the last 3 days, and (d) the glycosylated haemoglobin (HbAlc). A significant correlation $(P<0.01$ or $P<0.001$ ) was observed between the ischaemia-induced changes in axonal threshold and all of the parameters mentioned above (see Table 1). However, the highest regres- 
TABLE 1

CORRELATION BETWEEN ISCHAEMIA-INDUCED THRESHOLD CHANGES AND BLOOD GLUCOSE CONCENTRATIONS OR GLYCOSYLATED HAEMOGLOBIN

\begin{tabular}{|c|c|c|c|c|}
\hline & \multirow{2}{*}{$\begin{array}{l}\text { Actual blood } \\
\text { glucose }\end{array}$} & \multicolumn{2}{|c|}{ Mean blood glucose of ${ }^{a}$} & \multirow{2}{*}{$\begin{array}{l}\text { Glycosylated } \\
\text { haemoglobin }^{b}\end{array}$} \\
\hline & & $24 h$ & $72 \mathrm{~h}$ & \\
\hline $\begin{array}{l}\text { Threshold current } \\
\text { during ischaemia, } \\
5 \text { min after onset }\end{array}$ & $\begin{array}{l}r=0.70 \\
n=28 \\
P<0.001\end{array}$ & $\begin{array}{l}r=0.80 \\
n=28 \\
P<0.001\end{array}$ & $\begin{array}{l}r=0.72 \\
n=28 \\
P<0.001\end{array}$ & $\begin{array}{l}r=0.58 \\
n=20 \\
P<0.01\end{array}$ \\
\hline $\begin{array}{l}\text { Threshold current } \\
1 \text { min after an } \\
\text { ischaemic period } \\
\text { of } 10 \mathrm{~min}\end{array}$ & $\begin{array}{l}r=-0.64 \\
n=28 \\
P<0.001\end{array}$ & $\begin{array}{l}r=-0.85 \\
n=28 \\
P<0.001\end{array}$ & $\begin{array}{l}r=-0.82 \\
n=28 \\
P<0.001\end{array}$ & $\begin{array}{l}p=-0.66 \\
n=20 \\
p<0.01\end{array}$ \\
\hline
\end{tabular}

a Time period before examination.

b Determined within 4 weeks before examination.

sion coefficient was found for the mean blood glucose concentration over the last $24 \mathrm{~h}$ as illustrated in Fig. 3.

\section{Superexcitability}

Eleven diabetics ( 8 female, 3 male) were selected for their pronounced resistance to ischaemia. The post-spike changes in excitability of these patients were compared with a control group. Fig. 4 illustrates both the results of threshold tracking and of post-spike excitability determinations in these two populations. Part A illustrates the susceptibility to ischaemia. Controls and diabetics showed an increase in excitability starting within a few seconds after the onset of ischaemia. After 5 min the threshold fell
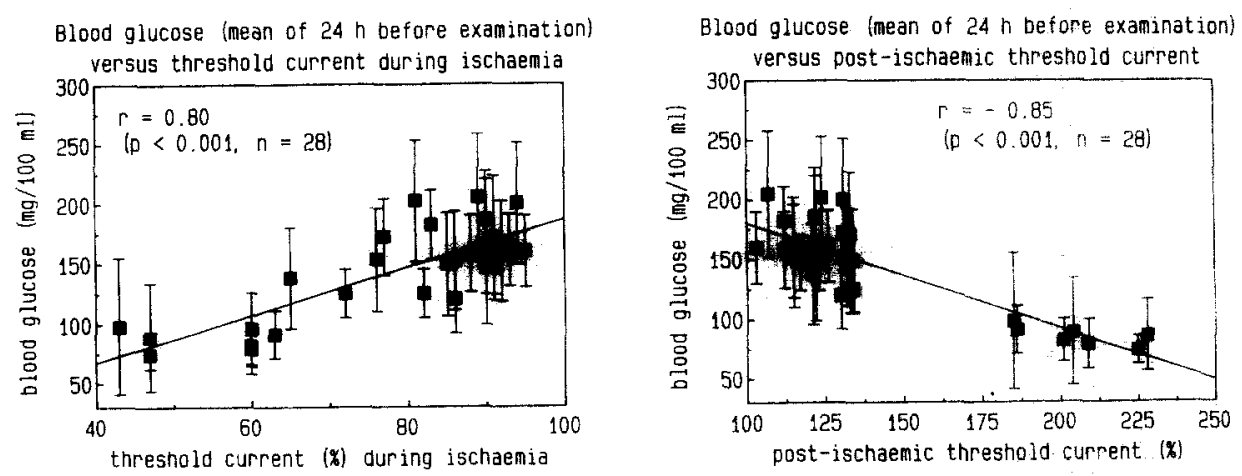

Fig. 3. Mean blood glucose concentrations (mean \pm SD) of the last $24 \mathrm{~h}$ before examination are plotted against the threshold current $(\%)$ during ischaemia ( 5 min after the onset) and after ischaemia ( 1 min after the end of $10 \mathrm{~min}$ period of ischaemia). $100 \%$ is the normalized value of threshold current in the preischaemic period. 
A

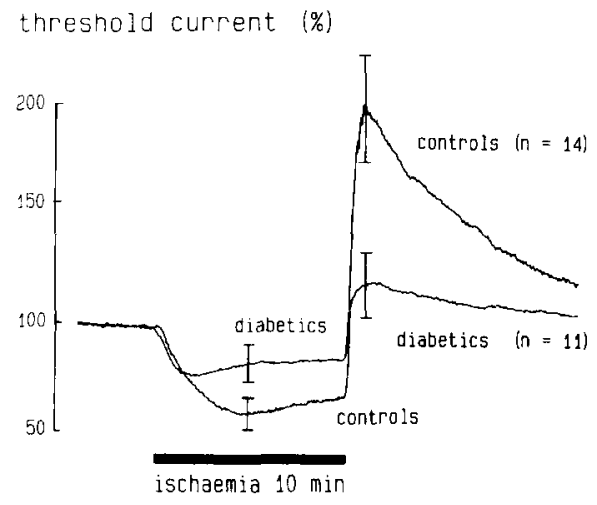

B

amplitude of test stimulus

$(\%)$

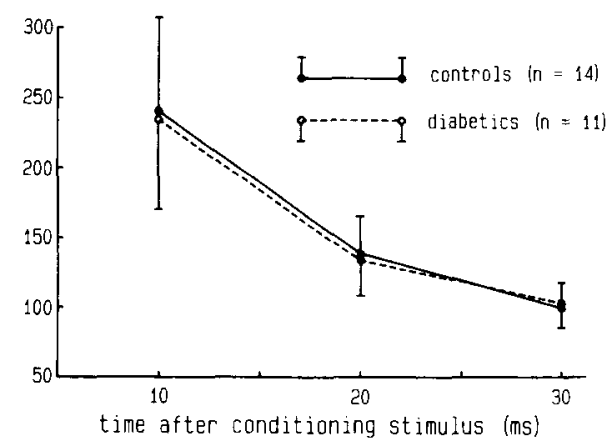

Fig. 4. A comparison between susceptibility to ischaemia and post-spike excitability. (A) Susceptibility to ischaemia. Changes in excitability of the peroneal nerve at the popliteal fossa before, during, and after $10 \mathrm{~min}$ of ischaemia were determined using the method of "threshold tracking". The figure shows averaged data from 14 control persons and 11 selected diabetics (mean \pm SD). The quantitative analysis was performed $5 \mathrm{~min}$ following the onset of ischaemia and $1 \mathrm{~min}$ after the release of the pressure cuff. (B) Changes in post-spike excitability. Data show the amplitude of a conditioned compound muscle action potential (CMAP) 10,20 , and $30 \mathrm{msec}$ after a supramaximal conditioning pulse. (100\% is the amplitude without a preceding stimulus.) No differences between controls and diabetics were found (mean $\pm S D$ ). The recording of superexcitability was performed on controls and diabetics illustrated in (A) shortly before the test for sensitivity to ischaemia.

to $59.1 \pm 7.1 \%$ (mean $\pm \mathrm{SD}, n=14$ ) of the pre-ischaemic level in controls and to $82.4 \pm 7.9 \%(n=11)$ in diabetics. Even more striking were the differences in the post-ischaemic decrease in excitability: threshold rose to about twice the pre-ischaemic level $(200.6 \pm 23.8 \%)$ in controls and only to $117.6 \pm 14.9 \%$ in diabetics. Consequently, a clear difference between controls and diabetics was found in respect of the susceptibility to ischaemia.

All the controls and diabetics illustrated in Fig. 4A were also tested for changes in the excitability of their peroneal nerves at the popliteal fossa in the period between 10 and $30 \mathrm{msec}$ following a supramaximal conditioning stimulus (see Methods; Fig. 1). These data were recorded $10-5 \mathrm{~min}$ before the ischaemic test. The observations are summarized in Fig. 4B. Both controls and diabetics showed a clear period of superexcitability; the averaged data do not differ significantly from each other.

\section{DISCUSSION}

The main aim of the present study was to explore whether axonal depolarization underlies the resistance to ischaemia in the peripheral nerves of diabetic subjects. Since direct recordings of membrane potential are not feasible in patients, an indirect method was used to reveal a possible decrease in resting potential. The method is based on the well known superexcitability of myelinated axons (Gilliat and Willison 1963; Bergmans 1970): the action potential of myelinated nerve fibres is followed by a long lasting after-depolarization (Barrett and Barrett, 1982) which lowers the threshold for a second 
spike 3-30 msec after a conditioning action potential. It has been shown that afterdepolarization and superexcitability are potential-sensitive. Both phenomena disappear when the membrane depolarizes, e.g., as a consequence of ischaemia, high extracellular $\mathrm{K}^{+}$concentration, and/or depolarizing current (Bergmans 1970; Stöh 1981; Barrett and Barrett 1982; Bergmans 1982; Bostock and Grafe 1985; Baker et al. 1987). The biophysical basis for the disappearance of post-spike superexcitability in depolarized fibres has been explained by Barrett and Barrett (1982). Depolarization reduced the input resistance of the fibres, and, therefore, reduced the depolarization of the internodal axon during an impulse. In lizard axons, the after-depolarization was reduced to zero by a depolarization of about $12 \mathrm{mV}$. Post-spike superexcitability is, therefore, a sensitive indicator of membrane potential changes, and this was shown in our experiments by its abolition after only $4 \mathrm{~min}$ of ischaemia (Fig. 1). Because superexcitability is so sensitive to depolarization, the absence of a significant difference between the post-spike excitability cycles of normals and diabetics (Fig. 4B) provides good evidence that membrane depolarization is not an important feature of diabetic axons.

The significant correlation of blood glucose concentrations or glycosylated haemoglobin (HbA1c) levels with ischaemia-related threshold changes supports and extends previous observations made with other electrophysiological methods (Steiness 1961b; Gregersen 1968; Horowitz and Ginsberg-Fellner 1979). The new method of threshold tracking is at least as sensitive for the metabolic alterations in diabetic nerves as ischaemia-induced changes in vibratory perception threshold, decrease in motor conduction velocity, or decline in sensory nerve compound action potential. However, in contrast to these other methods, threshold tracking can reveal statistically significant changes within only $5-10 \mathrm{~min}$ of ischaemia. Correlation coefficients as high as 0.85 between mean blood glucose and ischaemic threshold changes indicate that $70 \%$ or so of the variance in ischaemic sensitivity of the diabetics is accounted for by the relationship with blood glucose. The regression lines fall close to the expected data points for the controls (although their blood glucose was not measured in this study), reinforcing the evidence that blood glucose is the major determinant of ischaemic resistance. The best correlation in our study was found between the electrophysiological data and the mean blood glucose concentration over the last $24 \mathrm{~h}$ before examination. The correlation coefficients for the actual blood glucose concentration or the $\mathrm{HbAlc}$ values were lower. In rats made hyperglycaemic with streptozotocin or glucose injections, resistance to ischaemia has been seen as early as $2 \mathrm{~h}$ (Shirabe et al. 1988). A pressure cuff can be applied for $5 \mathrm{~min}$ as often as every $15 \mathrm{~min}$ to obtain consistent measurements of ischaemic sensitivity by threshold tracking, so this method should allow the time relationship between blood glucose and ischaemic resistance to be precisely defined.

The main reason for the changes in axonal excitability observed is thought to be changes in membrane potential: depolarization lowers threshold during ischaemia, and hyperpolarization reduces excitability in the post-ischaemic phase (Bergmans 1970). However, diabetics did not approach the low ischaemic threshold level of controls. In contrast, after about $3 \mathrm{~min}$ of ischaemia an increase in threshold was observed (see Figs. 2 and 4). This indicates another important factor opposed to the threshold lowering due to membrane depolarization. A likely candidate is extracellular acidosis 
due to anaerobic glycolysis. A decrease in $\mathrm{pH}$ is well known to reduce excitability (e.g., Bostock and Grafe 1985).

In conclusion, our data argue against the idea that the ischaemic resistance of axons in diabetics without neuropathy is due to membrane depolarization. They support the view that such nerves behave peculiarly during ischaemia due to increased substrate stores for anaerobic metabolism.

\section{ACKNOWLEDGEMENTS}

We would like to thank Ms. G. Hubert and Ms. C. Müler for technical and secretarial assistance, and Dr. E. Schmidbauer for his valuable help in the design and construction of the electronic equipment. This work was supported by the Wilhelm Sander Stiftung (P.G.) and the Medical Research Council (H.B.).

\section{REFERENCES}

Baker, M., H. Bostock, P. Grafe and P. Martius (1987) Function and distribution of three types of rectifying channel in rat spinal root myelinated axons. J. Physiol., 383: 45-67.

Barrett, E. F. and J.N. Barrett (1982) Intracellular recording from vertebrate myelinated axons: mechanism of the depolarizing afterpotential. J. Physiol., 323: 117-144.

Bergmans, J. (1970) The Physiology of Single Human Nerve Fibres. Vander, Louvain.

Bergmans, J. (1982) Modifications induced by ischemia in the recovery of human motor axons from activity. In: Culp, W.J. and J. Ochoa (Eds.), Abnormal Nerves and Muscles as Impulse Generators, Oxford University Press, London, pp. 419-442.

Bostock, H. and P. Grafe (1985) Activity-dependent excitability changes in normal and demyelinated rat spinal root axons. J. Physiol., 365: 239-257.

Das, P. K., G. M. Bray, A. J. Aguayo and M. Rasminsky (1976) Diminished ouabain-sensitive, sodium-potassium ATPase activity in sciatic nerves of rats with streptozotocin-induced diabetes. Exp. Neurol, 53: 285-288.

Dyck, P.J. (1989) Hypoxic neuropathy - does hypoxia play a role in diabetic neuropathy? The 1988 Robert Wartenberg Lecture. Neurology, 39: 111-118.

Gilliat, R. W. and R.G. Willison (1963) The refractory and supernormal periods of the human median nerve. J. Neurol. Neurosurg. Psychiat., 26: 136-147.

Greene, D. A., S. A. Lattimer and A.A.F. Sima (1988) Are disturbances of sorbitol, phosphoinositide, and $\mathrm{Na}^{+}-\mathrm{K}^{+}$-ATPase regulation involved in pathogenesis of diabetic neuropathy? Diabetes, 37: 688-693.

Gregersen, G. (1968) A study of the peripheral nerves in diabetic subjects during ischaemia. J. Neurol. Neurosurg. Psychiat., 31: 175-181.

Horowitz, S. H. and F. Ginsberg-Fellner (1979) Ischemia and sensory nerve conduction in diabetes mellitus. Neurology, 29: 695-704.

Jaramillo, J., N. Simard-Duquesne and D. Dvornik (1985) Resistance of the diabetic rat nerve to ischemic inactivation. Can. J. Physiol. Pharmacol., 63: 773-777.

Low, P. A. (1987) Recent advances in the pathogenesis of diabetic neuropathy. Muscle Nerve, 10: 121-128.

Low, P.A., K. Ward, J.D. Schmelzer and S. Brimijoin (1985) Ischemic conduction failure and energy metabolism in experimental diabetic neuropathy. Am.J. Physiol., 248: E457-E462.

Ludin, H. and W. Tackmann (1984) Polyneuropathien, Thieme, Stuttgart, New York.

Masson, E. A., S.E. Church, A. A. Woodcock, S.P. Hanley and A.J.M. Boulton (1988) Is resistance to ischaemic conduction failure induced by hypoxia? Diabetologia, 31: 762-765.

Newrick, P.G., A.J. Wilson, J. Jakubowski, A.J.M. Boulton and J.D. Ward (1986) Sural nerve oxygen tension in diabetes. Br. Med. J., 293: 1053-1054

Oh, S. J. (1984) Clinical Electromyography - Nerve Conduction Studies, University Park Press, Baltimore, MD. 
Parry, G. J. and H. Kohzu (1989) Studies of resistance to ischemic nerve conduction failure in normal and diabetic rats. J. Neurol. Sci., 93: 61-67.

Ritchie, J.M. (1985) A note on the mechanism of resistance to anoxia and ischaemia in pathophysiological mammalian myelinated nerve. J. Neurol. Neurosurg. Psychiat., 48: 274-277.

Shirabe, S., I. Kinoshita, H. Matsuo, H. Takashima, T. Nakamura, M. Tsujihata and S. Nagataki (1988) Resistance to ischemic conduction block of the peripheral nerve in hyperglycemic rats: An electrophysiological study. Muscle Nerve, 11: 582-587.

Steiness, I. (1959) Vibratory perception in diabetes during arrested blood flow to the limb. Acta Med. Scand., 163: 195-205.

Steiness, I. (1961a) Vibratory perception in non-diabetic subjects during ischaemia; with special reference to the conditions in hyperglycaemia; after carbohydrate starvation and after cortisone administration. Acta Med. Scand., 169: 17-26.

Steiness, I. (1961b) Influence of diabetic status on vibratory perception during ischaemia. Acta Med. Scand., 170: 319-338.

Stöhr, M. (1981) Modification of the recovery-cycle of human median nerve by ischemia. $J$. Neurol. Sci., 51: 171-180.

Strupp, M., P. Weigl, H. Bostock, K. Piwernetz, R. Renner and P. Grafe (1990) Is resistance to ischemia of motor axons in diabetic subjects due to membrane depolarization? Pflügers Arch, , 415: $\mathbf{R} 114$.

Thomas, P.K. and M.J. Brown (1987) Diabetic polyneuropathy. In: Dyck, P. J., P. K. Thomas, A. K. Asbury, A. I. Winegrad and D. Porte (Eds.), Diabetic Neuropathy, Saunders Company, Philadelphia, PA, pp. $56-65$.

Tuck, R. R., J. D. Schmelzer and P. A. Low (1984) Endoneurial blood flow and oxygen tension in the sciatic nerves of rats with experimental diabetic neuropathy. Brain, 107: 935-950.

Weigl, P., H. Bostock, P. Franz, P. Martius, W. Muller and P. Grafe (1989) Threshold tracking provides a rapid indication of ischemic resistance in motor axons of diabetic subjects. Electroenceph. Clin. Neurophysiol., 73: 369-371. 